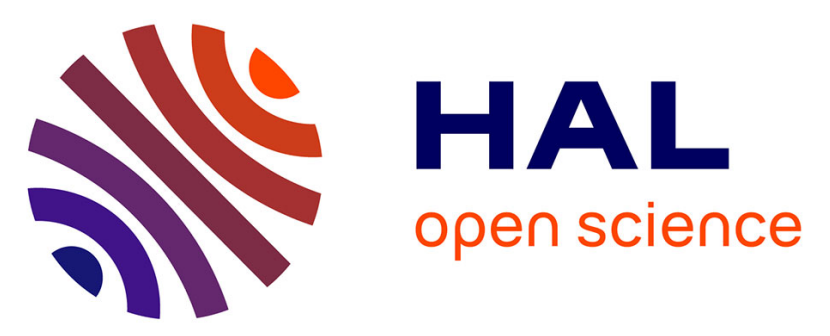

\title{
Electro-optic, thermo-optic, and dielectric properties of YCOB and Nd:YCOB crystals: comparative study
}

\author{
Mustapha Abarkan, Jean-Paul Salvestrini, Denis Pelenc, Marc Fontana
}

\section{To cite this version:}

Mustapha Abarkan, Jean-Paul Salvestrini, Denis Pelenc, Marc Fontana. Electro-optic, thermo-optic, and dielectric properties of YCOB and Nd:YCOB crystals: comparative study. Journal of the Optical Society of America B, 2005, 22 (2), pp.398-406. 10.1364/JOSAB.22.000398 . hal-00186010

\section{HAL Id: hal-00186010 https://hal.science/hal-00186010}

Submitted on 2 Dec 2021

HAL is a multi-disciplinary open access archive for the deposit and dissemination of scientific research documents, whether they are published or not. The documents may come from teaching and research institutions in France or abroad, or from public or private research centers.
L'archive ouverte pluridisciplinaire HAL, est destinée au dépôt et à la diffusion de documents scientifiques de niveau recherche, publiés ou non, émanant des établissements d'enseignement et de recherche français ou étrangers, des laboratoires publics ou privés.

\section{다)(1) $(5$}

Distributed under a Creative Commons Attribution - NonCommerciall 4.0 International 


\title{
Electro-optic, thermo-optic, and dielectric properties of YCOB and Nd:YCOB crystals: comparative study
}

\author{
Mustapha Abarkan and Jean Paul Salvestrini \\ Laboratoire Matériaux Optiques, Photonique et Systèmes, Centre National de la Recherche Scientifique (FRE CNRS 2304), \\ Université de Metz et Supélec, 2, Rue E. Belin 57070 Metz, France \\ Denis Pelenc \\ Laboratoire d'Electronique et de Technologie de l'Information, Département Optronique, Service Technologies des Composants \\ Optoélectroniques, Laboratoire Composants et Dispositifs Optoélectroniques, Commissariat à l'Energie Atomique, Centre de \\ Grenoble, 17 Rue des Martyrs, 38054 Grenoble Ce'dex 9, France
}

Marc D. Fontana

Laboratoire Matériaux Optiques, Photonique et Systèmes, Centre National de la Recherche Scientifique (FRE CNRS 2304), Université de Metz et Supélec, 2, Rue E. Belin 57070 Metz, France

\begin{abstract}
The electro-optic (EO), thermo-optic, and dielectric coefficients of pure as well as Nd-doped $\mathrm{YCa}_{4} \mathrm{O}\left(\mathrm{BO}_{3}\right)_{3}$ crystals are determined. The frequency and wavelength dependence of the EO coefficients were determined in the $0-150 \mathrm{MHz}$ and $0.5-1.3 \mu \mathrm{m}$ ranges, respectively. The analysis of the frequency dependences of both EO and dielectric properties provides the unclamped and clamped values of the whole set of coefficients. It appears that the acoustic contributions to the EO and dielectric properties are very low. Small differences in the properties found for the two crystals are attributed to the Nd doping ions. It is to be noticed that, surprisingly, the birefringence $\left(n_{X}-n_{Y}\right)$ was found to be independent of the temperature in the range $20-40{ }^{\circ} \mathrm{C}$.
\end{abstract}

\section{INTRODUCTION}

$\mathrm{YCa}_{4} \mathrm{O}\left(\mathrm{BO}_{3}\right)_{3}$ (YCOB) is a newly developed nonlinear optical crystal that has recently attracted wide interest because YCOB melts nearly congruently ${ }^{1-3}$ and can be pulled directly from the melt, which leads to the growth of large crystals. It was also found that the $\mathrm{Y}$ ion can be easily replaced by a $\mathrm{Nd}$ or $\mathrm{Yb}$ ion to form doped crystals of $\mathrm{Nd}: \mathrm{YCOB}$ and $\mathrm{Yb}: \mathrm{YCOB}$.

Self-conversion operation (emission of light at $1064 \mathrm{~nm}$ and second-harmonic generation, SHG) was recently achieved $^{4}$ in Nd:YCOB crystal. The integration of other optical functions, such as electro-optic (EO) modulation, requires extensive knowledge of the EO, thermo-optic (TO), and electrical properties. Unfortunately, until now, data available in the literature concern measurements of low-frequency values of the EO coefficients at the wavelength of $633 \mathrm{~nm}^{5,6}$ only.

In the present work $\mathrm{EO}, \mathrm{TO}$, and dielectric coefficients of pure as well as Nd-doped YCOB crystals are determined. We report, in Section 3, the frequency dependence of the EO coefficients in YCOB and Nd:YCOB crystals (the concentration $[\mathrm{Nd}] /([\mathrm{Nd}]+[\mathrm{Y}]$ ) is equal to 7 at. \% in the melt and 5 at. $\%$ in the crystal) within the frequency range of $0-150 \mathrm{MHz}$. The unclamped $r^{T}$ and clamped $r^{S}$ values of the EO coefficients, as well as the corresponding acoustic contributions, are thus determined in several optogeometrical configurations, and their dispersion in the visible and near-infrared ranges are measured. Then we present the temperature dependence of the spontaneous birefringences, from which we determine the values of the TO coefficient in the temperature range between $15{ }^{\circ} \mathrm{C}$ and $40^{\circ} \mathrm{C}$. We report, in Section 4 , the measurements of the frequency dispersion of the three dielectric permittivities in both YCOB and Nd:YCOB crystals. Finally, in Section 5 we discuss the origin of the EO properties and their variation between pure and Nd-doped crystals.

\section{CRYSTAL GROWTH AND LINEAR OPTICAL PROPERTIES OF YCOB AND Nd:YCOB CRYSTALS}

\section{A. Crystal Growth and Sample Preparation}

Because of their congruent melting, YCOB and Nd:YCOB can be grown from the melt by the Czochralski techniques. We have developed a growth process for single crystals of good optical quality by using an iridium crucible of $100-\mathrm{mm}$ diameter. Pulling rates in the range of $0.5-3 \mathrm{~mm} / \mathrm{h}$ were chosen, and the crucible rotation rate was chosen to keep the interface shape convex enough. Crystals were grown by pulling along the crystallographic $b$ axis direction of an oriented seed.

The thermal environment had to be carefully optimized with appropriate screens to avoid fractures during crystal cooling. The presence of some inclusions in the crystal 


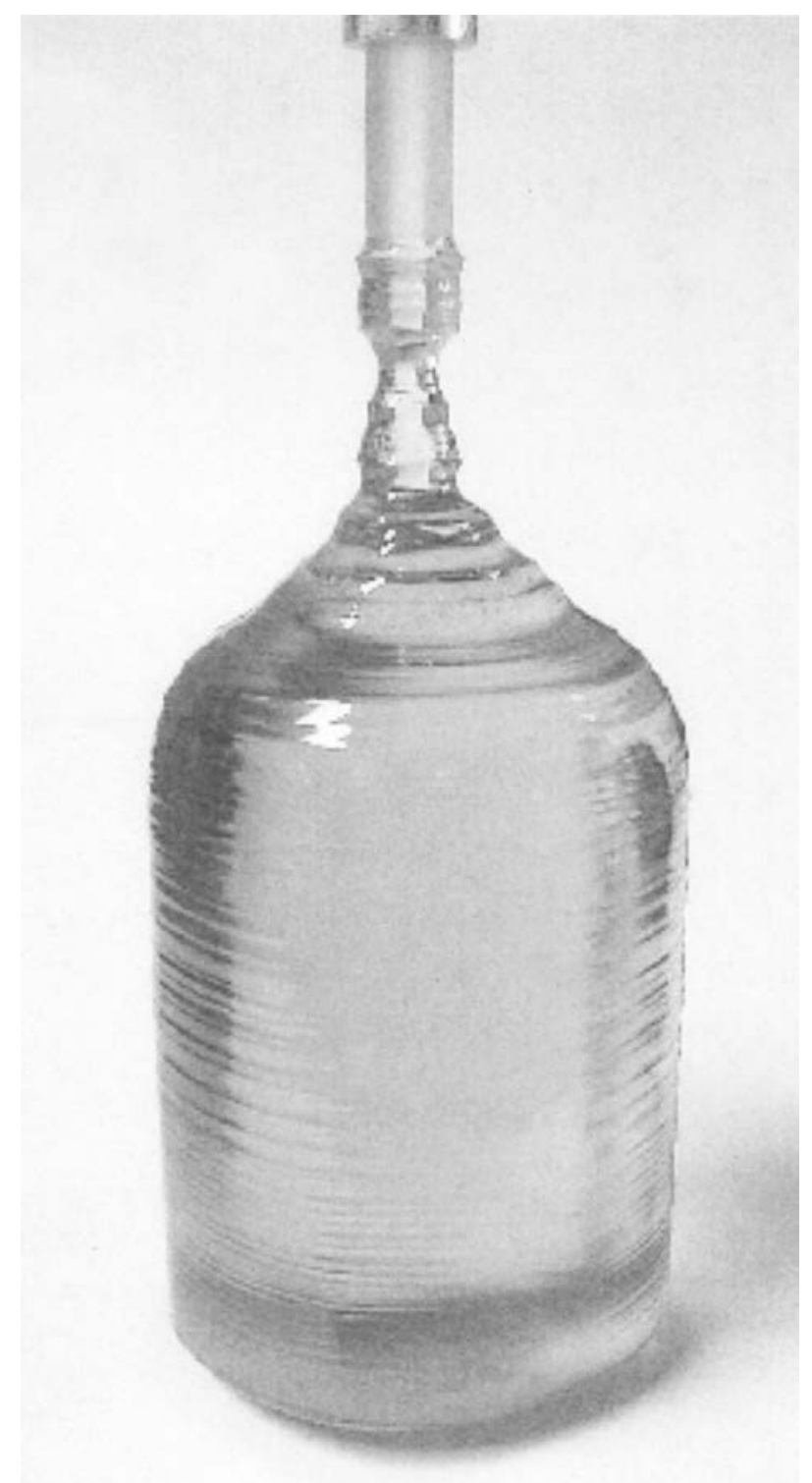

Fig. 1. Example of an as-grown Nd:YCOB crystal. Some growth striation is still visible inside the crystal.

was found to be linked to noise in the crystal diameter control loop, which used the crystal weight to monitor the crucible temperature. Therefore particular care was taken in the quality of the mechanical translation and rotation of the pulling head and in the crystal weight measurement in order to reduce the noise and the resulting diameter variations. Postgrowth annealing of the crystal was also necessary to stabilize the crystal and reduce the occurrence of fractures during sawing.

These process improvements finally led to the growth of crystals of good optical quality with dimensions reaching $50 \mathrm{~mm}$ in diameter and $120 \mathrm{~mm}$ in length, as can be seen in Fig. 1. However, some growth striations were still visible inside the crystal, revealing small index variations.

The crystals were cut with a multiwire saw with free $\mathrm{SiC}$ abrasive. A large sample with faces perpendicular to the crystallophysical axes $X, Y, Z$ was cut at the beginning. Subsequently slices with the required thickness were extracted from this sample.
The first step was to cut the planes perpendicular to the $Y$ direction. This particular axis being parallel to the $b$ axis in monoclinic biaxial crystals, it was possible to check the orientation accurately with an x-ray goniometer in a standard $\theta-2 \theta$ configuration. This led to a thick slab parallel to the $(X, Z)$ plane. Then the two perpendicular $X$ and $Z$ directions were oriented in the slab between cross polarizers. The assignment of $X$ and $Z$ indices to these directions was made after cutting, using an incident 1064-nm pulsed laser and measuring the incidence angle at which SHG was observed, as described below.

The block with three planes perpendicular to the crystallographic axes was cut in rectangular slices of thickness $1.675 \mathrm{~mm}$ with the multiwire saw and then polished to optical quality. Mechanochemical polishing slurry was used for finishing, on polyurethane pads. Figure 2 shows a block and the oriented pieces that were cut and polished along the crystallographic axis in a YCOB slab for this study.

The orientation of the slices was checked with a SHG experimental setup. A pulsed laser emitting at $1064 \mathrm{~nm}$ with a linear, vertical polarization was directed onto the slice to be characterized. This slice was mounted on a goniometric plate with its $Y$ direction parallel to the polarization axis and along the rotation axis. The $Y$ direction had been unambiguously assigned by $\mathrm{x}$-ray diffraction previously.

The angle configuration for which SHG was observed allowed the unambiguous assignment of the indices of the slice axis: For $Z$ slices, an SHG interaction of type I can be excited in the $Z X$ plane. The corresponding incidence

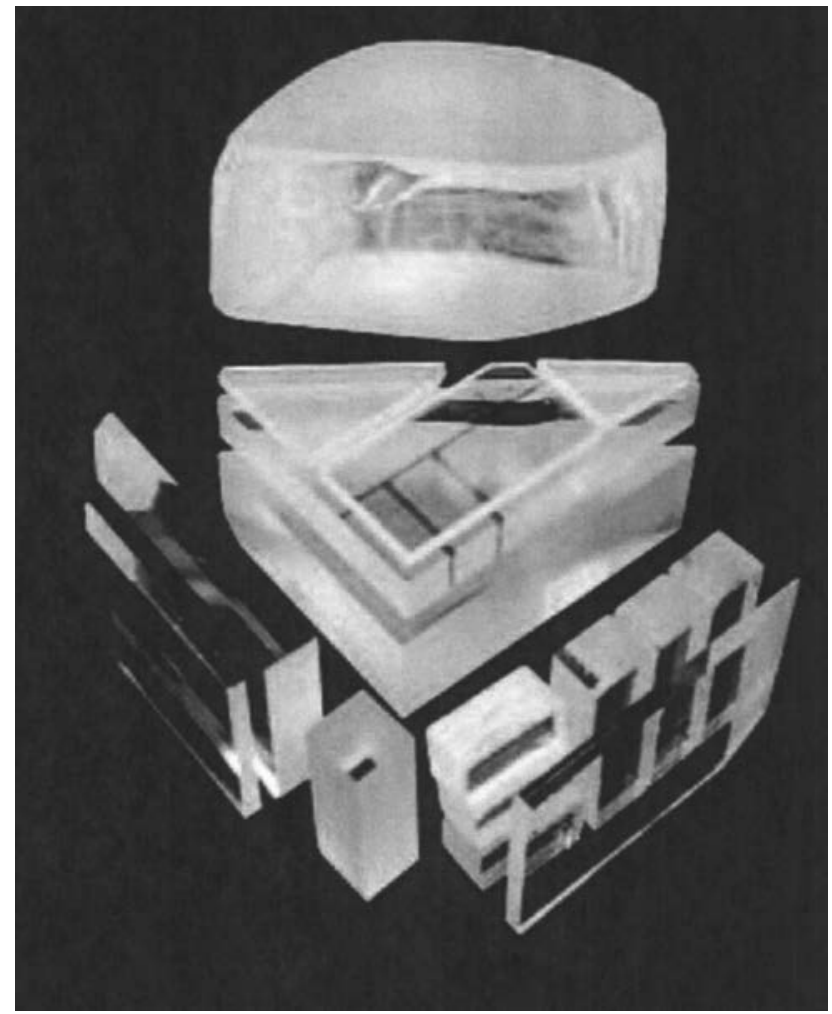

Fig. 2. Block and the oriented pieces that were cut and polished along the crystallographic axis in a YCOB slab. 
Table 1. Refractive Indices of Pure YCOB from the Sellmeier Equation ${ }^{8}$

\begin{tabular}{ccccccc}
\hline $\begin{array}{c}\text { Wavelength } \\
(\mathrm{nm})\end{array}$ & $n_{X}$ & \multicolumn{1}{c}{$n_{Y}$} & \multicolumn{1}{c}{$n_{Z}$} & $n_{1}$ & $n_{2}$ & $n_{3}$ \\
\hline 514 & 1.6744 & 1.7317 & 1.7514 & 1.7116 & 1.7414 & 1.7023 \\
633 & 1.6584 & 1.7143 & 1.7333 & 1.6946 & 1.7237 & 1.6857 \\
815 & 1.6461 & 1.7006 & 1.7188 & 1.6813 & 1.7096 & 1.6727 \\
1064 & 1.6376 & 1.6903 & 1.708 & 1.6717 & 1.6991 & 1.6633 \\
1321 & 1.6321 & 1.6829 & 1.7003 & 1.6651 & 1.6915 & 1.6569 \\
\hline
\end{tabular}

angle is $\sim 57^{\circ} .{ }^{7}$ Slices for which no SHG was observed under $57^{\circ}$ of incidence were rotated in their mount by $90^{\circ}$ around their normal axis, so that the rotation axis of the goniometer was along $Z$. The $X$ nature of the normal to these samples was checked by the observation of SHG under an incidence angle of $\sim 80^{\circ}$, corresponding to the type I $X Y$ interaction. ${ }^{7}$

Moreover, the measurement of the two symmetric incident angles $i_{1}$ and $i_{2}$ leading to SHG and the corresponding refracted angles $r_{1}$ and $r_{2}$ allowed us to estimate the misorientation $\alpha$ of $Z$ (or $X$ ) with respect to the normal axis of the slice, these angles being linked by the simple relations

$$
r_{1}=\theta+\alpha, \quad r_{2}=\theta-\alpha
$$

\section{B. Linear Optical Properties}

YCOB and Nd:YCOB are monoclinic biaxial crystals with point group symmetry $m .^{1}$ The orientation between the optical main axes $(X, Y, Z)$ and the crystallographic axes $(a, b, c)$ has been determined ${ }^{8}$ to be

$$
b \| Y, \quad(a, Z)=24.7^{\circ}, \quad(c, X)=13.4^{\circ} .
$$

It is to be noted that this orientation does not depend on the wavelength of the optical beam in the range 400-2200 $\mathrm{nm}$. The main values of the refractive indices $n_{X}, n_{Y}$ and $n_{Z}$ corresponding to the principal dielectric axes $X, Y$, and $Z$ were determined at different wavelengths by use of the Sellmeier equation given by Segonds et al. ${ }^{8}$ in the case of the YCOB crystal. These values, as well as the values of the effective refractive indices $n_{1}, n_{2}$, and $n_{3}$ (needed in Section 3), defined by

$$
\begin{aligned}
& \frac{1}{n_{1}^{2}}=\frac{1}{2 n_{Z}^{2}}+\frac{1}{2 n_{X}^{2}}, \\
& \frac{1}{n_{2}^{2}}=\frac{1}{2 n_{Y}^{2}}+\frac{1}{2 n_{Z}^{2}}, \\
& \frac{1}{n_{3}^{2}}=\frac{1}{2 n_{X}^{2}}+\frac{1}{2 n_{Y}^{2}},
\end{aligned}
$$

are presented in Table 1. Unfortunately, to our knowledge the linear optical properties of Nd:YCOB are still unavailable.

\section{ELECTRO-OPTICAL AND THERMO- OPTICAL MEASUREMENTS}

\section{A. Electro-Optic Coefficients and Related Configurations}

As mentioned above, YCOB and Nd:YCOB crystals belong to the monoclinic point group $m$. The EO tensor therefore has ten nonzero independent components, which are $r_{11} r_{21}, r_{31}, r_{51}, r_{42}, r_{62}, r_{13}, r_{23}, r_{33}$, and $r_{53}$. In this study we have characterized the EO coefficients $r_{42}, r_{62}$, $r_{51}$, and $r_{53}$ and the effective EO coefficients, defined by several linear combinations of the components of the EO tensor, $r_{a 1}, r_{a 2}, r_{c 1}$, and $r_{c 2}$ which are defined as follows:

$$
\begin{aligned}
r_{a 1} & =r_{11}-\frac{n_{Y}}{n_{X}} r_{31}, & r_{a 2} & =r_{11}-\frac{n_{Z}}{n_{X}} r_{21}, \\
r_{c 1} & =r_{33}-\frac{n_{X}}{n_{Z}} r_{23}, & r_{c 2} & =r_{33}-\frac{n_{Y}}{n_{Z}} r_{13} .
\end{aligned}
$$

The EO coefficients $r_{a 1}$ and $r_{a 2}$ were measured in configurations (see Fig. 3) for which the light beam propagates along the principal $Z$ axis and $Y$ axis, respectively, and for which the electrical field is applied along the $X$ axis. The EO coefficients $r_{c 1}$ and $r_{c 2}$ were determined for the light beam propagating along the principal $Y$ axis and $X$ axis, respectively, and for an applied electric field along
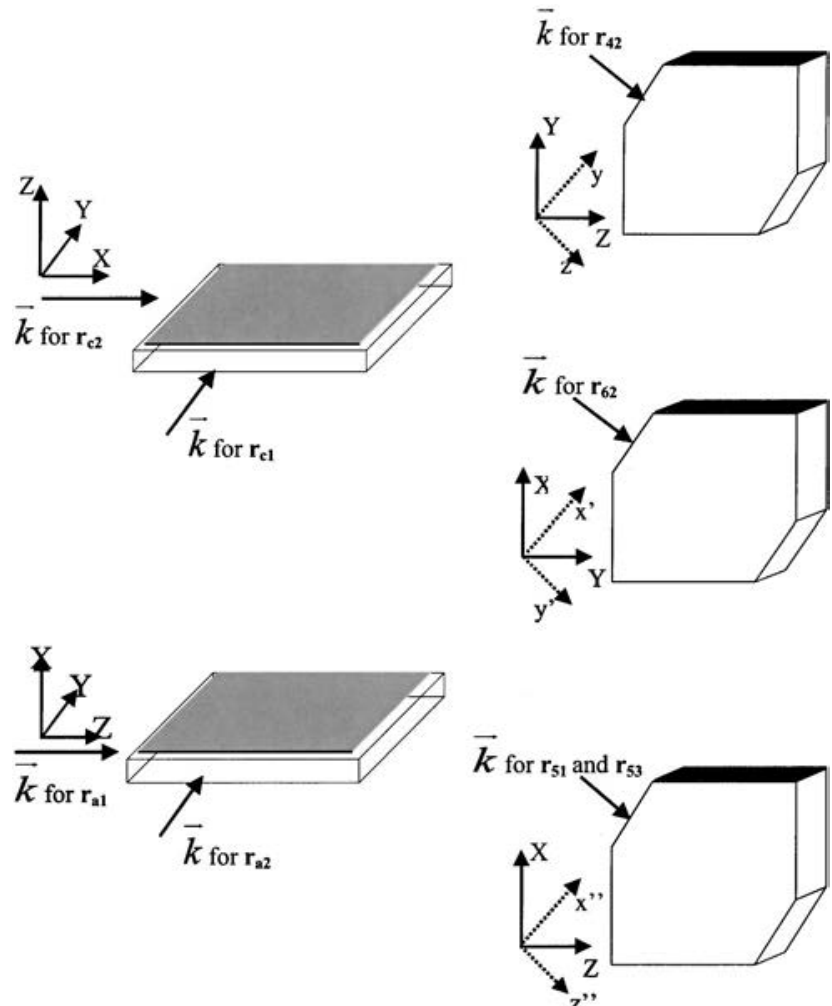

Fig. 3. Description of the sample configurations used for the measurements of the EO coefficients $r_{a 1}, r_{a 2}, r_{c 1}, r_{c 2}, r_{42}, r_{62}$, $r_{51}$, and $r_{53}$ in the YCOB and Nd:YCOB crystals. $X, Y$, and $Z$ are the crystallophysical axes. The impinging light beam is linearly polarized at $45^{\circ}$ with respect to the neutral lines of the crystal. In the case of the EO coefficients $r_{51}$ and $r_{53}$, the gold electrodes were deposited on the $X$ and $Z$ faces; $\vec{k}$, beam propagation direction. 
the $Z$ axis (see Fig. 3). Thus, the field-induced birefringences in the $(X, Z),(X, Y)$, and $(Y, Z)$ planes are given, respectively, by

$$
\begin{aligned}
& \Delta n_{X Z}\left(E_{X}\right)=-\frac{n_{X}^{3} r_{a 1} E_{X}}{2}, \\
& \Delta n_{X Z}\left(E_{Z}\right)=-\frac{n_{Z}^{3} r_{c 2} E_{Z}}{2}, \\
& \Delta n_{X Y}\left(E_{X}\right)=-\frac{n_{X}^{3} r_{a 2} E_{X}}{2}, \\
& \Delta n_{Y Z}\left(E_{Z}\right)=-\frac{n_{Z}^{3} r_{c 1} E_{Z}}{2} .
\end{aligned}
$$

The principal EO coefficients $r_{42}, r_{62}, r_{51}$, and $r_{53}$, called skew EO coefficients, are obtained when the optical beam is propagating at $45^{\circ}$ with respect to the two principal dielectric axes. In the case of the coefficient $r_{42}$, the light beam propagation is at $45^{\circ}$ from the $Y$ and $Z$ axes, and the electric field is applied along the $Y$ axis. For the coefficient $r_{62}$, the electric field remains applied along the $Y$ axis, and the light beam propagates at $45^{\circ}$ with respect to the axes $X$ and $Y$. For the two other coefficients $r_{51}$ and $r_{53}$, the configuration is such that the beam propagation direction is at $45^{\circ}$ from the $X$ and $Z$ axes and the applied electric field direction is along the $X$ axis and the $Z$ axis, respectively. These configurations thus need special cuts (see Fig. 3).

Finally, the field-induced birefringences in the $(X, y)$, $\left(Y, x^{\prime \prime}\right)$ and $\left(x^{\prime}, y^{\prime}\right)$ planes are given, respectively by

$$
\begin{aligned}
\Delta n_{X y}\left(E_{Y}\right) & =-\frac{n_{2}^{3} r_{42} E_{Y}}{2}, \\
\Delta n_{Y x^{\prime \prime}}\left(E_{X}\right) & =-\frac{n_{1}^{3} r_{51} E_{X}}{2}, \\
\Delta n_{Y x^{\prime \prime}}\left(E_{Z}\right) & =-\frac{n_{3}^{3} r_{53} E_{Z}}{2}, \\
\Delta n_{x^{\prime} y^{\prime}}\left(E_{Y}\right) & =-\frac{n_{2}^{3} r_{62} E_{Y}}{2} .
\end{aligned}
$$

The $x$ axis ( $y$ axis) is obtained by a rotation of the $X$ axis ( $Y$ axis) by $45^{\circ}$ around the $Y$ axis ( $X$ axis), and the refractive indices $n_{1}, n_{2}$ and $n_{3}$ are defined in Eqs. (3). The axes $x^{\prime}\left(x^{\prime \prime}\right)$ and $y^{\prime}\left(z^{\prime \prime}\right)$ are obtained by a rotation of the axes $X$ and $Y(X$ and $Z)$, respectively, by $45^{\circ}$ around the $Z$ axis ( $Y$ axis).

\section{B. Experimental Setup and Method}

The experimental setup used to measure the EO coefficients was based on the Sénarmont arrangement (see Fig. 4).

The crystal followed by a $\lambda / 4$ plate was located between a linear polarizer and an analyzer. The polarizer was set to yield the laser light polarized at $45^{\circ}$ to the principal axes of the crystal. The $\lambda / 4$ plate axes were also set at $45^{\circ}$ to the crystal axes, while the analyzer was oriented at

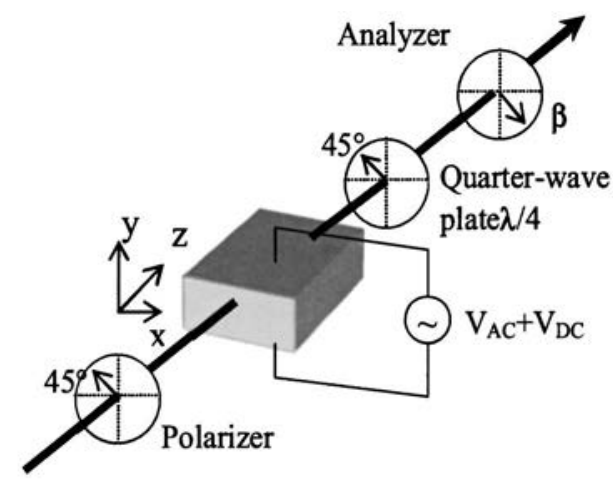

Laser beam

(a)

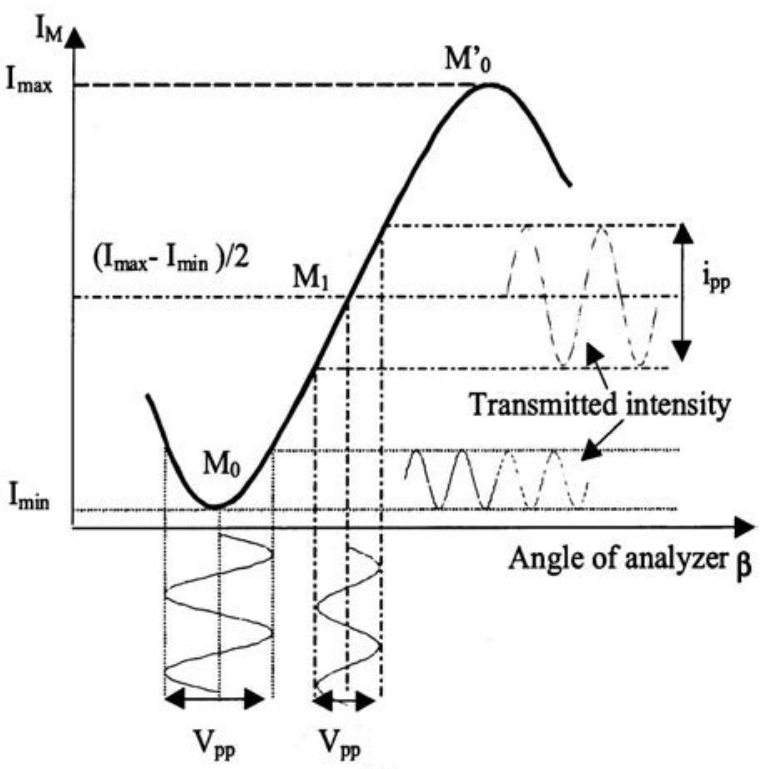

(b)

Fig. 4. (a) Sénarmont arrangement used for the EO measurements. (b) Optical transmission versus the analyser angle $\beta$. $M_{0}$, minimum transmission point for which the output optical signal has a frequency twice as large as the frequency of the applied electric field; $M_{1}, 50 \%$ transmission point yielding the linear replica of the ac voltage.

an azimuthal angle $\beta$. The transfer function of the light intensity transmitted through the setup can be written as $^{9}$

$$
\mathrm{T}=\frac{1-\gamma \sin (\Gamma-2 \beta)}{2}
$$

where $\beta$ is the angular position of the analyzer and $\Gamma$ is the phase shift introduced by the EO crystal, whereas

$$
\gamma=\frac{I_{\max }-I_{\min }}{I_{\max }+I_{\min }}
$$

is the contrast, which is usually considered a constant parameter. The phase shift $\Gamma_{E}$ and the birefringence $\Delta n(E)$ induced by the electric field $E$ are each linked to the other by the relationship 


$$
\Gamma_{E}=\frac{2 \pi L}{\lambda} \Delta n(E)=\frac{\pi}{\lambda} L n_{\mathrm{eff}}^{3} r_{\mathrm{eff}} E,
$$

where $L$ is the length of the crystal along the beam propagation direction, $\lambda$ is the wavelength, and $n_{\text {eff }}$ and $r_{\text {eff }}$ are the effective refractive index and the EO coefficient, respectively.

We used the time response method. ${ }^{9}$ In particular, we determined the clamped (or constant-strain) $r^{S}$ coefficient and the unclamped (or constant-stress) $r^{T}$ coefficient. The method consists in measuring the EO crystal's response time to a fast voltage step. The instantaneous optical response after the voltage step (rising time of few nanoseconds) leads to the clamped coefficient $r^{S}$, whereas the optical response observed after several microseconds' duration of the square voltage pulse leads to the coefficient $r^{T}$.

The voltage-induced intensity variation is maximum at the linear part of the transfer function. At this working point the time dependence of the variation of the transmitted beam intensity $\Delta i(t)$ induced by the applied voltage $\Delta V(t)$ yields ${ }^{9}$ the time response of the EO crystal under test,

$$
\Delta i(t)=\frac{\pi n_{\mathrm{eff}}^{3} L I_{0}}{2 \lambda d} r_{\mathrm{eff}}(t) \otimes \Delta V(t),
$$

where $\otimes$ is the convolution operator, $d$ is the thickness of the crystal efficient, $I_{0}=I_{\max }-I_{\min }$ represents the total intensity shift of the transfer function, and $r_{\text {eff }}(t)$ is the value of the $\mathrm{EO}$ coefficient. The frequency dispersion of the EO coefficients can be derived from the ratio of the quantities $\Delta i(\nu)$ and $\Delta V(\nu)$, which are obtained by the $Z$ transformation of the measured signals $\Delta i(t)$ and $\Delta V(t)$. We get

$$
r_{\mathrm{eff}}(\nu)=\frac{2 \lambda d}{\pi n_{\mathrm{eff}}^{3} I_{0} L} \frac{\Delta i(\nu)}{\Delta V(\nu)} .
$$

We have shown ${ }^{9}$ that this technique allows us to obtain the frequency dispersion of the EO coefficient from dc up to at least $150 \mathrm{MHz}$; this maximum depends only on the rising time of the voltage pulse.

\section{Results}

The method, described just above, was used to obtain the frequency dispersion of the various EO coefficients $r_{a 1}$, $r_{a 2}, r_{c 1}, r_{c 2}, r_{42}, r_{62}, r_{51}$, and $r_{53}$ in the YCOB and Nd:YCOB crystals. It is to be noted that the values of the co-

Table 2. Dimensions of the YCOB and Nd:YCOB Samples $^{a}$

\begin{tabular}{lcc}
\hline & YCOB & Nd:YCOB \\
EO Coefficient & $X \times Y \times Z(\mathrm{~mm})$ & $X \times Y \times Z(\mathrm{~mm})$ \\
\hline$r_{a 1}, r_{a 2}$ & $1.675 \times 19 \times 22$ & $1.675 \times 16.5 \times 11.8$ \\
$r_{c 1}, r_{c 2}$ & $18.5 \times 18.5 \times 1.675$ & $10.5 \times 16.5 \times 1.675$ \\
$r_{42}, r_{62}$ & $1.675 \times 19 \times 18.5$ & $1.675 \times 11.8 \times 11.8$ \\
$r_{51}, r_{53}$ & $9 \times 1.675 \times 8$ & $11.2 \times 1.675 \times 11.8$ \\
\hline
\end{tabular}

${ }^{a} X, Y$, and $Z$ are the principal dielectric axes of the crystal as shown in Fig. 3 .

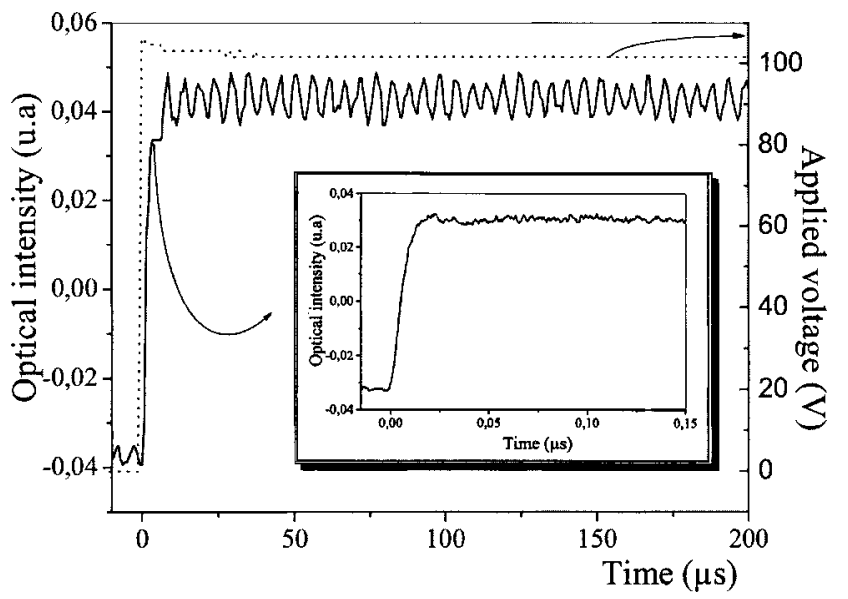

Fig. 5. Plots versus time of the applied voltage and the optical signal at different time scales for the configuration involving the EO coefficient $r_{c 2}$ in the YCOB crystal. The wavelength of the measurements was $633 \mathrm{~nm}$.

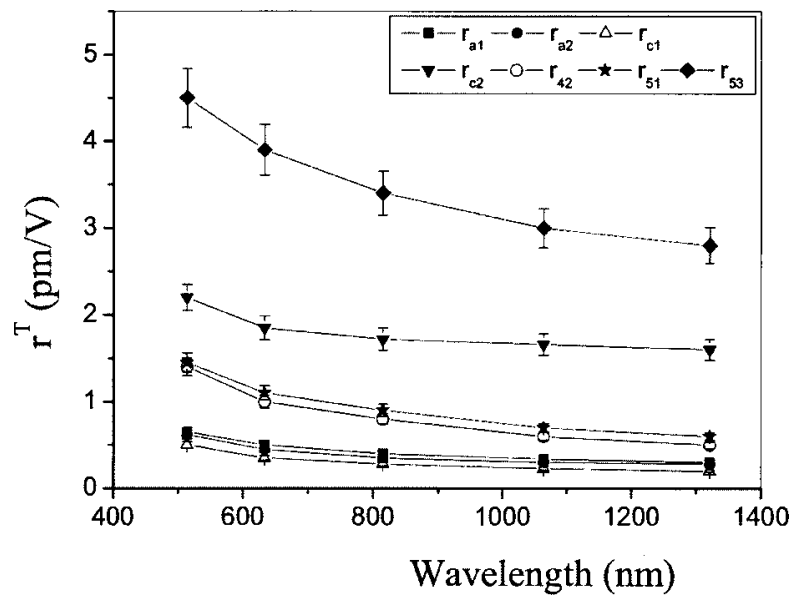

Fig. 6. Wavelength dependence of the unclamped $\left|r_{\text {eff }}^{T}\right|$ EO coefficients in the YCOB crystal.

efficients obtained by this technique and given in the following are absolute values. The signs of the coefficients were not determined.

Several samples of each crystal were cut and polished, and gold electrodes were deposited on them. We summarize in Table 2 the characteristics of the samples that were used as well as their dimensions.

To determine the frequency dependence of the EO coefficients, we recorded both the applied voltage $\Delta V(t)$ and the optical signal $\Delta i(t)$. The frequency dependence is then obtained from Eqs. (10) and (11). As an example, Fig. 5 shows the recording of both the applied voltage and the optical signal (for two different time scales) measured for the configuration involving the EO coefficient $r_{c 2}$ in YCOB crystal. In the long-time range the optical signal oscillates with a period that corresponds to the main piezoelectric frequency resonance. The oscillations tend to disappear for times longer than several tens of microseconds. They do not exist for time shorter than $0.2 \mu \mathrm{s}$, since the acoustic waves need more time to propagate across the crystal. The signal at short times is nearly constant and can be compared to the one recorded for long times, above several tens of microseconds. The shift between these values yields the acoustic contribution ${ }^{10} r^{a}$. 
These measurements were achieved for the whole set of EO coefficients and for several wavelengths: $514 \mathrm{~nm}(\mathrm{Ar}$ laser), $633 \mathrm{~nm}$ (HeNe laser), $815 \mathrm{~nm}$ (laser diode), 1064 and $1321 \mathrm{~nm}$ (Nd:YAG laser). The complete set of results, in particular the wavelength dependence of the unclamped and clamped EO coefficients, is reported in Figs. 6 and 7 for YCOB and in Figs. 8 and 9 for Nd:YCOB crystals. Since the values of the refractive indices of $\mathrm{Nd}$ :YCOB are unknown, Fig. 8 exhibits the wavelength dependences of the product $\left|n_{\text {eff }}^{3} r_{\text {eff }}\right|$ instead of those of $\left|r_{\text {eff }}\right|$. As we can see on these figures, the clamped and unclamped values of the EO coefficients are very close, indicating a small acoustic contribution $r^{a}$ to the EO properties.

As is shown in Table 3, our results are generally in a good agreement with the previous measurements reported at $633 \mathrm{~nm}$ by other authors. ${ }^{5,6}$ Nevertheless, the results reported in Table 3 reveal some discrepancies between the values obtained by Adams and Ebbers ${ }^{6}$ and our values for the coefficients exhibiting low values. By contrast, the values of the large coefficient $r_{c 2}$ are in fair agreement, within the experimental errors. The measurements of Yin et $a l .^{5}$ have shown that the largest coefficient of the YCOB crystal is $r_{33}$. This is inconsistent

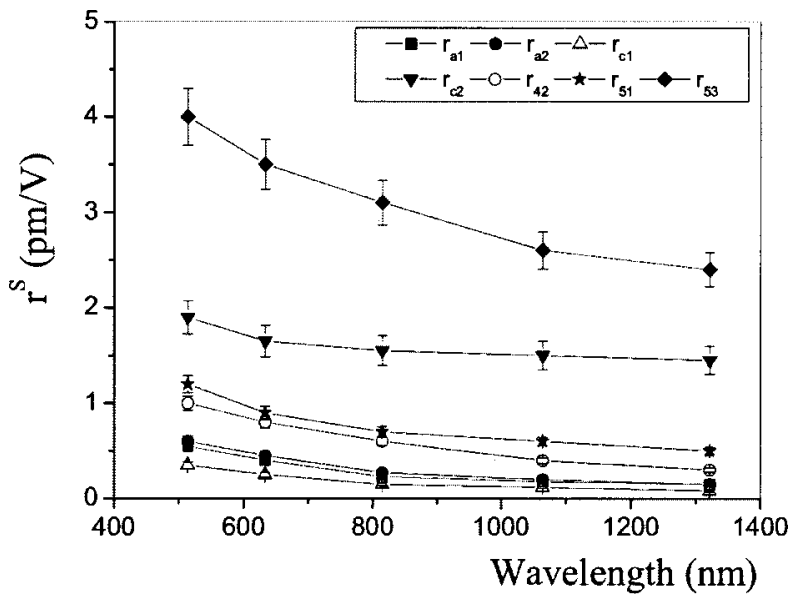

Fig. 7. Wavelength dependence of the clamped $\left|r_{\text {effl }}^{S}\right|$ EO coefficients in the YCOB crystal.

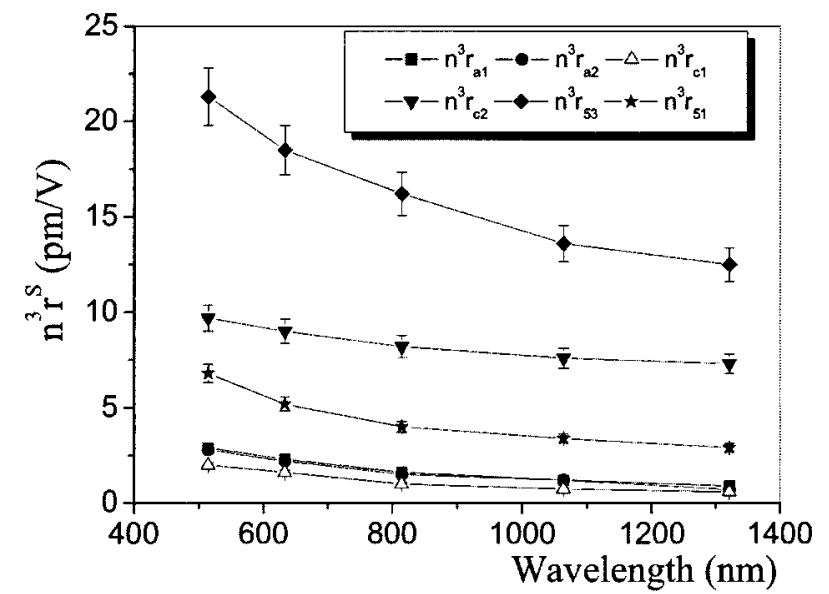

Fig. 8. Wavelength dependence of the unclamped $\left|n_{\text {eff }}^{3} r_{\text {eff }}^{T}\right|$ EO coefficients in the Nd:YCOB crystal.

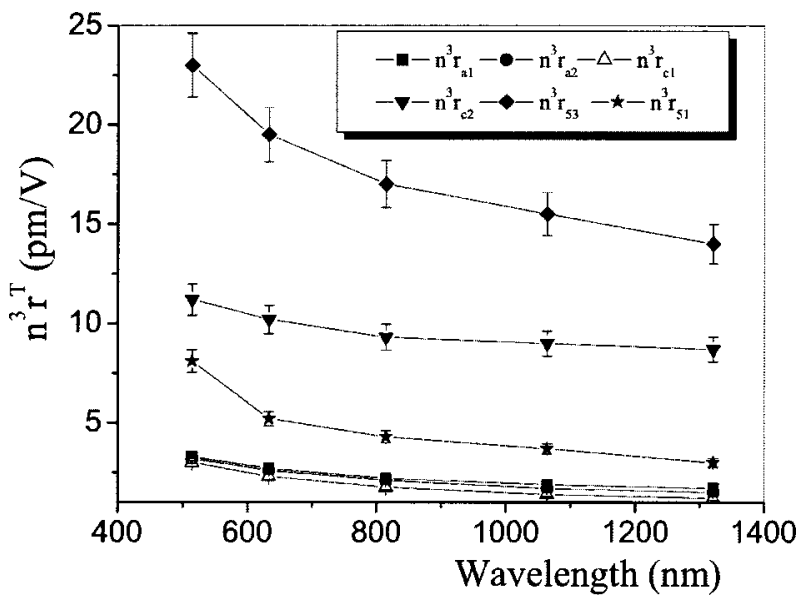

Fig. 9. Wavelength dependence of the clamped $\left|n_{\text {eff }}^{3} r_{\text {eff }}^{S}\right|$ EO coefficients in the Nd:YCOB crystal.

Table 3. Comparison of Unclamped Values of $\left|n_{\text {eff }}^{3} r_{\text {eff }}^{T}\right|$ and $\left|r_{\text {eff }}^{T}\right|$ EO Coefficients for the YCOB Crystal at $632.8 \mathrm{~nm}$

\begin{tabular}{cccc}
\hline $\begin{array}{c}\text { Effective EO Coefficient } \\
\left(\mathrm{pm} \mathrm{V}^{-1}\right)\end{array}$ & Ref. 5 & Ref. 6 & Present results \\
\hline$\left|n_{X}^{3} r_{a 1}\right|$ & - & $<0.2$ & $2.3 \pm 0.2$ \\
$\left|n_{X}^{3} r_{a 2}\right|$ & - & $<0.82$ & $2.1 \pm 0.2$ \\
$\left|n_{Z}^{3} r_{c 1}\right|$ & - & $<0.52$ & $1.85 \pm 0.2$ \\
$\left|n_{Z}^{3} r_{c 2}\right|$ & - & $10.8 \pm 1.4$ & $9.7 \pm 1$ \\
$\left|r_{42}\right|$ & 0.8 & - & $1 \pm 0.1$ \\
$\left|r_{62}\right|$ & 0.4 & - & $0.5 \pm 0.05$ \\
$\left|r_{51}\right|$ & 0.9 & - & $0.9 \pm 0.1$ \\
$\left|r_{53}\right|$ & 4.1 & - & $3.5 \pm 0.3$ \\
\hline
\end{tabular}

with our results and the results of Adams and Ebbers, ${ }^{6}$ since we have found that the value of $r_{\mathrm{c} 2}$, which is a combination of $r_{13}$ and $r_{33}$, is much larger than the value of $r_{c 1}$, which is a combination of $r_{23}$ and $r_{33}$. These two effective coefficients should be nearly equal if the coefficient $r_{33}$ were the largest. The discrepancy between the EO measurements of Yin et $a l .{ }^{5}$ on the one hand and the measurements of Adams and Ebbers ${ }^{6}$ or our results on the other hand, could be attributed to the nonnegligible piezoelectric effect of the YCOB crystal, which in an interferometric setup can introduce a large systematic error ${ }^{11}$ into the results. Indeed, the additional phase shift coming from the deformation of the crystal under the applied electric field can yield a shift of the EO coefficient.

We report in Table 4 the values of the unclamped $n_{\text {eff }}^{3} r_{\text {eff }}^{T}$ and clamped $n_{\text {eff }}^{3} r_{\text {eff }}^{S}$ EO coefficients measured in both YCOB and Nd:YCOB crystals at a wavelength of $1064 \mathrm{~nm}$. From Tables 3 and 4 we can derive three features: (i) The EO properties are highly anisotropic in both crystals; (ii) the unclamped value is systematically larger than the corresponding clamped value for each EO coefficient in both crystals; (iii) the value of the EO coefficient in Nd-doped crystal is always slightly larger than the value in pure YCOB crystal. Even if $\left|n_{\text {eff }}^{3} r_{\text {eff }}\right|$ is actually considered in the comparison, the large difference that is due to $\mathrm{Nd}$ doping cannot be really attributed to a change in the refractive index. All these remarks will be interpreted in Section 5. 


\section{Thermo-Optic Effect}

To measure the TO coefficients of the two crystals, we used the point (Fig. 4) at which the transmitted intensity is minimum, $M_{0}$ (i.e., $I=I_{\min }$ ). An alternating electric field of frequency $\nu$ applied to the EO crystal generates an optical signal modulated at the double frequency $2 \nu$. The double-frequency signal is lost as a variation of temperature is introduced and can be recovered if the analyzer is rotated by an given angle $\beta$. The variation of the birefingence induced by the TO effect is given by ${ }^{12}$

$$
\frac{\mathrm{d} \Delta n}{\mathrm{~d} T}=\frac{\lambda}{\pi L} \frac{\mathrm{d} \beta}{\mathrm{d} T}
$$

Table 4. Comparison of Unclamped $\left|n_{\mathrm{eff}}^{3} \boldsymbol{r}_{\mathrm{eff}}^{T}\right|$ and Clamped $\left|n_{\text {eff }}^{3} r_{\text {eff }}^{S}\right|$ EO Coefficients Measured in YCOB and Nd:YCOB Crystals at $1064 \mathrm{~nm}$

\begin{tabular}{crr}
\hline $\begin{array}{c}\text { Effective EO Coefficient } \\
\left(\mathrm{pm} \mathrm{V} \mathrm{V}^{-1}\right)\end{array}$ & \multicolumn{1}{c}{ YCOB } & Nd:YCOB \\
\hline$\left|n_{X}^{3} r_{a 1}^{T}\right|$ & $1.5 \pm 0.15$ & $1.9 \pm 0.2$ \\
$\left|n_{X}^{3} r_{a 2}^{T}\right|$ & $1.3 \pm 0.1$ & $1.7 \pm 0.2$ \\
$\left|n_{Z}^{3} r_{c 1}^{T}\right|$ & $1.1 \pm 0.1$ & $1.4 \pm 0.1$ \\
$\left|n_{Z}^{3} r_{c 2}^{T}\right|$ & $8.0 \pm 0.6$ & $9 \pm 0.7$ \\
$\left|n_{1}^{3} r_{51}^{T}\right|$ & $2.9 \pm 0.3$ & $3.7 \pm 0.3$ \\
$\left|n_{3}^{3} r_{53}^{T}\right|$ & $14.1 \pm 1$ & $15.5 \pm 1.1$ \\
& & \\
$\left|n_{X}^{3} r_{a 1}^{S}\right|$ & $0.9 \pm 0.1$ & $1.2 \pm 0.1$ \\
$\left|n_{X}^{3} r_{a 2}^{S}\right|$ & $0.9 \pm 0.1$ & $1.2 \pm 0.1$ \\
$\left|n_{Z}^{3} r_{c 1}^{S}\right|$ & $0.6 \pm 0.1$ & $0.9 \pm 0.1$ \\
$\left|n_{Z}^{3} r_{c 2}^{S}\right|$ & $7 \pm 0.5$ & $7.8 \pm 0.6$ \\
$\left|n_{1}^{3} r_{51}^{S}\right|$ & $3 \pm 0.3$ & $3.4 \pm 0.3$ \\
$\left|n_{3}^{3} r_{53}^{S}\right|$ & $13 \pm 1$ & $13.6 \pm 1$ \\
\hline
\end{tabular}

Table 5. Absolute Values of TO Coefficients in YCOB and Nd:YCOB Crystals ${ }^{a}$

\begin{tabular}{lcc}
\hline TO Coefficient $\left(10^{-6} \mathrm{~K}^{-1}\right)$ & YCOB & Nd:YCOB \\
\hline$\left|\frac{\mathrm{d} \Delta n_{Y Z}}{\mathrm{~d} T}\right|$ & $59 \pm 2$ & $61 \pm 2$ \\
$\left|\frac{\mathrm{d} \Delta n_{X Z}}{\mathrm{~d} T}\right|$ & $2.6 \pm 0.1$ & $2.6 \pm 0.1$ \\
$\left|\frac{\mathrm{d} \Delta n_{X Y}}{\mathrm{~d} T}\right|$ & $0 \pm 0.1$ & $0 \pm 0.1$ \\
\hline
\end{tabular}

${ }^{a}$ Measured near room temperature at a wavelength of $1321 \mathrm{~nm}$

Table 6. Unclamped $\epsilon^{T}$ and Clamped $\epsilon^{S}$ Dielectric Constants in YCOB and Nd:YCOB Crystals ${ }^{a}$

\begin{tabular}{crr}
\hline Dielectric Constant & \multicolumn{1}{c}{ YCOB } & \multicolumn{1}{c}{ Nd:YCOB } \\
\hline$\epsilon_{\mathrm{X}}^{\mathrm{T}}$ & $8.7 \pm 0.7$ & $9.4 \pm 0.7$ \\
$\epsilon_{\mathrm{X}}^{\mathrm{S}}$ & $8.5 \pm 0.7$ & $9 \pm 0.7$ \\
$\epsilon_{\mathrm{Y}}^{\mathrm{T}}$ & $11.6 \pm 0.9$ & $11.9 \pm 0.9$ \\
$\epsilon_{\mathrm{Y}}^{\mathrm{S}}$ & $10.7 \pm 0.8$ & $11.4 \pm 0.9$ \\
$\epsilon_{\mathrm{Z}}^{\mathrm{T}}$ & $7.9 \pm 0.6$ & $10.1 \pm 0.8$ \\
$\epsilon_{\mathrm{Z}}$ & $7.7 \pm 0.6$ & $9.7 \pm 0.8$ \\
\hline
\end{tabular}

${ }^{a}$ Measured near room temperature.
The measurements consist in recording the analyzer angle $\beta$ as a function of the temperature. The slope of the curve $\beta$ versus temperature, from Eq. (12), provides the TO coefficient. These measurements were carried out for the two crystals under study for each principal spontaneous birefringence and at a wavelength of 1321 $\mathrm{nm}$. The results are reported in Table 5. As is seen in this table, an unexpected zero value was found for the TO coefficient related to the birefringence $\Delta n_{X Y}$ in both YCOB and Nd:YCOB crystals. This indicates that the refractive indices $n_{X}$ and $n_{Y}$ should have the same temperature dependence, at least in the range of our measurements $\left(20-40{ }^{\circ} \mathrm{C}\right)$.

\section{DIELECTRIC MEASUREMENTS}

To complete our set of characterizations, we measured the frequency dependence of the three relative dielectric permittivities $\epsilon_{X}, \epsilon_{Y}$, and $\epsilon_{Z}$ of the YCOB and Nd:YCOB crystals. Using the same samples as above, we obtain by means of a HP4192 impedance analyzer the dependences of various coefficients within a frequency range of $1 \mathrm{kHz}$ to $13 \mathrm{MHz}$.

The dependences exhibit many narrow peaks originating from the acoustic resonances. For both crystals the frequency value of the first resonance is larger in the case of $\epsilon_{Y}$ than in the case of $\epsilon_{Z}$ and $\epsilon_{X}$. This is likely due to the difference between the velocity of the acoustic waves along the three axes, since the three samples used have the same thickness. The anisotropy of the sound velocity in both crystals is rather large, since we can estimate that the velocity is ten times higher along the $Y$ axis than along the two other axes. The low-frequency (i.e., below the first resonance frequency) values of the dielectric constant corresponds to the unclamped value. In contrast, the high-frequency (above the last resonance frequency) value of the dielectric constant corresponds to the clamped value. These unclamped and clamped values of the three dielectric constants are reported in Table 6 for the both crystals.

This relatively large anisotropy is also present in the values of the EO coefficients (Table 4), TO properties (Table 5), and in the dielectric permittivity (Table 6) as well. For both crystals the largest value of the dielectric permittivity is obtained along the $Y$ axis. We can also see in Table 6 that the values of the dielectric constants in the $\mathrm{Nd}$ :YCOB crystal are slightly larger than those in the YCOB crystal. These differences between the two crystals do not depend on frequency and are especially great in the case of the dielectric permittivity measured along the $Z$ axis.

The dissipation factor $\tan \delta$ corresponding to the ratio of the imaginary part of the dielectric permittivity to its real part is low (less than 0.05 ), indicating very low electrical losses in both crystals.

\section{DISCUSSION}

Now let us discuss the origin of the EO properties in YCOB, their frequency dependence, and their change with Nd doping. All EO coefficients are smaller than 0.6 $\mathrm{pm} \mathrm{V}^{-1}$ (at the wavelength of $1064 \mathrm{~nm}$ ) except for $r_{53}$ and 
$r_{c 2}$, which are equal to $3.0 \mathrm{pm} \mathrm{V}^{-1}$ and $1.5 \mathrm{pm} \mathrm{V}^{-1}$, respectively. These last EO coefficients involve the modulation of the refractive indices by the electric field applied along the $Z$ axis.

We recall that the EO coefficient $r^{T}$ in an unclamped crystal results from the superposition of three contributions ${ }^{13}$ as follows. The electronic contribution $r^{e}$ arises from the direct modulation of electrons by the electric field. The ionic part $r^{i}$ comes from the modulation of electrons via the lattice. The acoustic contribution $r^{a}$ is an indirect EO process resulting from a combination of EO and piezoelectric effects. Within the EO process, the deformation modulates the refractive index through the elasto-optic effect and thus yields the additional contribution $r^{a}$ to $r^{T}$ at low frequencies; $r^{S}=r^{e}+r^{i}$ is the true EO coefficient measured in a clamped crystal. Whereas $r^{a}$ is determined from the difference between $r^{T}$ and $r^{S}$, $r^{e}$ can be evaluated from the nonlinear coefficient $d .^{13}$ Both pure and Nd-doped YCOB crystals exhibit in each EO coefficient a difference $r^{a}$ between the unclamped $r^{T}$ and the $r^{S}$ values that is not larger than $0.2 \mathrm{pm} \mathrm{V}^{-1}$.

Furthermore, the electronic part $r^{e}$ is small $^{3} \quad(\leqslant 1$ $\mathrm{pm} \mathrm{V}^{-1}$, deduced from the value of the largest nonlinear coefficient of YCOB, which is $d_{32}$ ). Therefore the relatively large EO coefficients $r_{c 2}$ and $r_{53}$ originate mainly from the ionic contribution. These large values can be attributed to oxygen octahedra centered on $\mathrm{Y}^{3+}$ and $\mathrm{Ca}^{2+}$ ions and linked by planar $\mathrm{BO}_{3}$ triangles. Indeed, it is well known that oxygen octahedra have large ionic (and electronic) polarizabilities and are responsible for strong EO (and nonlinear optic) properties in oxydic crystals such as $\mathrm{KNbO}_{3}, \mathrm{LiNbO}_{3}$ or $\mathrm{BaTiO}_{3} .{ }^{14}$

The EO coefficients are still larger in Nd-doped YCOB than in pure YCOB crystals, and the difference comes from the change in the ionic part that is due to Nd doping. In a simple description, $r^{i}$ results from the product of the polarization-optic coefficient and the dielectric susceptibility. The change in dielectric permittivity $\epsilon^{S}$ that is due to $\mathrm{Nd}$ doping is insufficient to explain the variation in the EO coefficient $r^{S}$. Furthermore, the anisotropy in the EO properties is stronger than in the dielectric coefficient. Therefore the enhancement of EO coefficients in Nd-doped YCOB crystal is due mainly to a change of the polarization-optic coefficient and is linked to a change in the ionic structure especially in the oxygen octahedra. As the $\mathrm{Nd}^{3+}$ ion has a larger radius than the $\mathrm{Y}^{3+}$ ion, the partial substitution of $\mathrm{Nd}^{3+}$ for $\mathrm{Y}^{3+}$ ions leads to an increase in the volume of oxygen octahedra, ${ }^{5}$ and thus to a larger deformability, and subsequently to a larger EO coefficient.

\section{CONCLUSION}

We have studied the electro-optic (EO), thermo-optic (TO), and dielectric properties of the YCOB and Nd:YCOB crystals. We have especially determined the frequency and wavelength dependences of the EO coefficients. The analysis of the frequency dependences of both EO and dielectric permittivity properties provides the unclamped and clamped values of the whole set of coefficients. The results show some differences in the properties between both crystals, which can be attributed to the $\mathrm{Nd}$ doping ions. Our work shows that the robust mechanical and thermomechanical nature of the YCOB crystal associated with relatively large EO coefficients within a large frequency range, a low dielectric permittivity, and a very weak TO coefficient could lead to applications in largeaperture, high-average-power, near-infrared $Q$ switches. ${ }^{15}$

\section{ACKNOWLEDGMENTS}

This study was undertaken within the framework of the network CMDO (Cristaux Massifs et Dispositifs Optiques), devoted to the study of bulk crystals and optical devices, with the support of the France National Center of Scientific Research. The authors thank Germano Montemezzani and Gérard Aka for helpful discussions.

*Present address, Center d'études Universitaires de Taza, Université Sidi Mohammed Ben Abdallah, BP 1323 Taza-Gare, Morocco.

\section{REFERENCES}

1. M. Iwai, T. Kobayashi, H. Furuya, Y. Mori, and T. Sasaki, "Crystal growth and optical characterization of rare-earth (Re) calcium oxyborate $\operatorname{ReCOB}(\mathrm{Re}=\mathrm{Y}$ or $\mathrm{Gd})$ as new nonlinear optical material,” Jpn. J. Appl. Phys. Part 1 36, 276279 (1997).

2. D. Vivien, G. Aka, A. Kahn-Harari, A. Aron, F. Mougel, J. M. Benitez, B. Ferrand, R. Klein, G. Kugel, N. LeNain, and M. Jacquet, "Crystal growth and optical properties of rare earth calcium oxoborates," J. Cryst. Growth 237, 621-628 (2002).

3. Q. Ye and B. H. T. Chai, "Crystal growth of YCOB and its orientation,” J. Cryst. Growth 197, 228-235 (1999).

4. M. C. Richardson, D. A. Hammons, J. Eichenholz, B. C. H. Chai, Q. Ye, W. K. Jang, and L. Shah, "YCOB lasers," J. Korean Phys. Soc. 37, 633-639 (2000).

5. X. Yin, J. Wang, J. Wei, and H. Gang, "Measurement of electro-optic coefficients of low-symmetry YCOB," Opt. Lasers Eng. 37, 643-649 (2002).

6. J. J. Adams and C. A. Ebbers, "Linear electro-optic properties of YCOB," Opt. Lett. 28, 1469-1471 (2003).

7. F. Mougel, "Les oxoborates de calcium et de terres rares (TR) $\mathrm{Ca}_{4} \mathrm{TRO}\left(\mathrm{BO}_{3}\right)_{3}$. Une nouvelle famille de matriaux fonctions multiples pour l'optique: croissance cristalline, proprits non linaires et laser," Ph.D. thesis (University of Paris 06, Paris, 1999).

8. P. Segonds, B. Boulanger, J. P. Feve, B. Menaert, J. Zaccaro, G. Aka, and D. Pelenc, "Linear and nonlinear optical properties of the monoclinic YCOB crystal," J. Opt. Soc. Am. B 21, 765-769 (2004).

9. M. Abarkan, J. P. Salvestrini, M. Aillerie, and M. D. Fontana, "Frequency dispersion of electro-optical properties over a wide range by means of time-response analysis," Appl. Opt. 42, 2346-2353 (2003).

10. A. R. Johnston, "The strain-free electro-optic effect in single crystal barium titanate," Appl. Phys. Lett. 7, 195-198 (1965).

11. J. Zaccaro, J. P. Salvestrini, A. Ibanez, P. Ney, and M. Fontana, "Electric-field frequency dependence of Pockels coefficients in 2-amino-5-nitropyridium dihydrogen phosphate organic-inorganic crystals," J. Opt. Soc. Am. B 17, 427-432 (2000).

12. M. Aillerie, M. D. Fontana, F. Abdi, C. Carabatos-Nedelec, N. Theofanous, and G. E. Alexakis, "Influence of the temperature-dependent spontaneous birefringence in the 
electro-optic measurements of $\mathrm{LiNbO}_{3}$, J. Appl. Phys. 65, 2406-2408 (1989).

13. J. P. Salvestrini, M. D. Fontana, B. Wyncke, and F. Brehat, "Comparative measurements of the frequency dependence of electro-optical and dielectric coefficients in inorganic crystals," Nonlinear Opt. 17, 271-280 (1997).

14. M. D. Fontana, K. Laabidi, B. Jannot, M. Maglione, and P.
Jullien, "Relationship between electro-optic, vibrational and dielectric properties in $\mathrm{BaTiO}_{3}$," Solid State Commun. 92, 827-830 (1994).

15. J. P. Salvestrini, M. Abarkan, and M. D. Fontana, "Comparative study of nonlinear optical crystals for electro-optic $Q$-switching of laser resonator," Opt. Mater. 26, 449-458 (2004). 